\title{
Efficient Methods of Doppler Processing for Coexisting Land and Weather Clutter
}

\author{
Çag̃atay Candan and A. Özgür Yılmaz \\ Middle East Technical University (METU) \\ Ankara, Turkey. \\ ccandan@metu.edu.tr, aoyilmaz@metu.edu.tr
}

\begin{abstract}
The joint suppression of returns from land and weather clutter is required in many radar applications. Although the optimal method of land-weather clutter suppression is known, the solution is usually not practical to implement. In this paper, we propose a method that employs rank-1 and rank-2 approximations on the weather clutter correlation matrix to obtain suboptimal but simpler detectors which are easier to implement. The performance of the proposed detectors is compared with the optimal detector and some other detectors commonly used for clutter suppression.
\end{abstract}

Index Terms-MTI, Doppler Processing, Weather Clutter

\section{INTRODUCTION}

We consider the problem of clutter cancellation for pulse Doppler radar systems when the received signal contains clutter return due to reflections from both land and weather systems. The problem studied frequently occurs in air surveillance radar systems. For such systems, the echo received may have components due to target signal, land clutter and return from dense clouds with non-zero velocity. Especially surveillance operation in rain or fog can be severely compromised by the weather clutter if its effect is not properly cancelled.

The stationary clutter (land clutter) cancellation problem is a well studied in the literature [1]. Moving target indicator (MTI) and Doppler processing techniques with DFT banks or optimized filterbanks have been studied in depth along with the optimal methods based on clutter correlation. Cancellation of weather clutter has been less elaborated. In [1], a simple MTI operation, whose null is at the Doppler frequency of weather clutter, is suggested. In [2], the performance of MTI for weather clutter cancellation is studied and its performance is compared with the optimal method under various conditions. It has been noted that the suppression performance of MTI is sufficient under some scenarios, but in some others it falls behind the moving target detector (MTD) filterbanks with optimized filters, [3].

In this paper, we re-examine the problem studied in [2] and propose a novel method approximating the optimal operation. With the proposed method, an approximate yet close to the optimal solution is achieved with realizable computational requirements.

The paper is organized as follows. In Section 2, we describe the problem, the clutter models and the optimal solution. In Section 3, we describe the MTI approach, whitening approach, and the proposed approximations to the optimal method. In
Section 4, we present numerical comparisons of the methods under various operational scenarios and finally we present the conclusions.

\section{PROBLEM DESCRIPTION}

A train of $N$ pulses are used to decide the presence of a target in a range cell. The vector $\mathbf{r}$ is assumed to contain the I/Q samples of the signal represented with the complex baseband representation:

$$
\mathbf{r}=\alpha \mathbf{s}+\sqrt{E_{l}} \mathbf{c}_{l}+\sqrt{E_{w}} \mathbf{c}_{w}+\sqrt{N_{o}} \mathbf{w} .
$$

In equation (1), the vector $\mathbf{s}$ is the desired signal denoting the return from the target, the vectors $\mathbf{c}_{l}$ and $\mathbf{c}_{w}$ are clutter return vectors due to land and weather systems, respectively. The vector $\mathrm{w}$ represents thermal noise and the entries of this vector are independent identically distributed circularly symmetric complex Gaussian random variables with zero mean and unit variance. The parameters $\alpha, E_{l}, E_{w}, N_{o}$ are scalars to represent the power of each term.

The vector $\mathbf{s}$ is assumed to be a deterministic quantity in the form $\left[1 W W^{2} \ldots W^{N-1}\right]$ where $W=e^{-j w_{t}}$. The parameter $w_{t}$ corresponds to the Doppler frequency of the target (in radians) after modulo PRF (pulse repetition frequency) reduction. The goal is to detect the deterministic signal, which is the complex exponential vector, in the presence of random disturbance due to clutter and thermal noise.

The auto-correlation of land clutter and weather clutter vectors $\mathbf{c}_{l}, \mathbf{c}_{w}$ are assumed to be in the form

$$
\mathbf{r}_{c}(k)=\rho^{k^{2}} \exp \left(\frac{-j 2 \pi f_{w} k}{\operatorname{PRF}}\right) \text {. }
$$

In (2), $f_{w}$ is the Doppler frequency (in Hertz) of the clutter object. For land clutter, $f_{w}$ is equal to zero and for weather clutter it is proportional to the radial velocity of the clouds. The correlation parameter $\rho$ is frequently used in radars with antenna scanning modulation. It is based on the assumption that clutter motion is insignificant in comparison with the motion of the antenna during the coherent processing interval. The parameter $\rho$ is related with the parameters of the radar system such as angular speed of the rotating antenna, PRF etc.

Under these conditions, the input SNR (for each received sample) becomes $\mathrm{SNR}^{\text {in }}=|\alpha|^{2} /\left(E_{l}+E_{c}+N_{o}\right)$. The goal of MTI or Doppler processing system is to increase the sample SNR by jointly processing $N$ samples of the vector $\mathbf{r}$. 
A linear combination operation of the received samples can be expressed as $\mathbf{w}^{H} \mathbf{r}$. Here $\mathbf{w}$ is the linear combination vector which is a column vector of length $N$. After linear combination, the SNR at the output becomes

$$
\mathrm{SNR}^{\text {out }}=|\alpha|^{2} \frac{\mathbf{w}^{H} \mathbf{S ~ s}^{H} \mathbf{w}}{\mathbf{w}^{H} \mathbf{R} \mathbf{w}} .
$$

Here the matrix $\mathbf{R}$ denotes the auto-correlation matrix of the clutter and thermal noise terms. More explicitly the ith row and kth column of matrix $\mathbf{R}$ is

$$
\mathbf{R}(i, k)=E_{l} r_{c l}(i-k)+E_{w} r_{c w}(i-k)+N_{o} \delta(i-k) .
$$

Here the terms $r_{c l}$ and $r_{c w}$ refer to land and weather clutter auto-correlations whose definitions are given in (2).

When $\mathbf{s}$ is a deterministic parameter, the components of $\mathrm{SNR}^{\text {out }}$ in (3) are totally deterministic. The vector $\mathbf{w}$ maximizing the ratio in (3) is the generalized eigenvector of matrix $\mathbf{R}$ and rank-1 matrix $\mathbf{s s}^{H}$. The optimal vector is given by $\mathbf{w}_{\text {opt }}=\mathbf{R}^{-1} \mathbf{s}$ [1], [4]. The optimal weight vector can be written as follows:

$$
\begin{aligned}
\mathbf{w}_{o p t} & =\mathbf{R}^{-1} \mathbf{s} \\
& =(E_{l} \mathbf{R}_{\mathbf{c}_{\mathbf{l}}}+\underbrace{E_{w} \mathbf{D}_{\mathbf{f}_{\mathbf{w}}} \mathbf{R}_{\mathbf{c}_{1}} \mathbf{D}_{\mathbf{f}_{\mathbf{w}}}^{\mathbf{H}}}_{\mathbf{R}_{\mathbf{w}}}+N_{o} \mathbf{I})^{-1} \mathbf{s} \\
& =\frac{1}{N_{o}}\left(\frac{E_{l}}{N_{o}} \mathbf{R}_{\mathbf{c}_{1}}+\frac{E_{w}}{N_{o}} \mathbf{D}_{\mathbf{f}_{\mathbf{w}}} \mathbf{R}_{\mathbf{c}_{\mathbf{l}}} \mathbf{D}_{\mathbf{f}_{\mathbf{w}}}^{\mathbf{H}}+\mathbf{I}\right)^{-1} \mathbf{s} .
\end{aligned}
$$

In (5), the matrix $\mathbf{R}_{\mathbf{c}_{1}}$ denotes the normalized auto-correlation matrix for land clutter. The weather clutter matrix $\left(\mathbf{R}_{\mathrm{w}}\right)$ is represented in terms of $\mathbf{R}_{\mathbf{c}_{1}}$ through prior and posterior multiplication by $\mathbf{D}_{f_{w}}$. The matrix $\mathbf{D}_{f_{w}}$ is a diagonal matrix with diagonal entries $\left[1, W, W^{2}, \ldots W^{N-1}\right]\left(W=e^{-j 2 \pi f_{w} / P R F}\right)$.

We note that the land clutter correlation matrix and weather clutter correlation matrix are similarity transformations of each other. Therefore both matrices have the same eigenvalues. We denote the eigen decomposition of land clutter correlation matrix as $\mathbf{R}_{\mathbf{c}_{1}}=\mathbf{E} \boldsymbol{\Lambda} \mathbf{E}^{H}$. Here $\mathbf{E}$ is the matrix whose columns are the eigenvectors of $\mathbf{R}_{\mathbf{c}_{1}}$ and $\boldsymbol{\Lambda}$ is a diagonal matrix with associated eigenvalues on the diagonal. Using the eigendecomposition in (5), we get

$\mathbf{w}_{o p t}=\frac{1}{N_{o}}\left(\frac{E_{l}}{N_{o}} \mathbf{E} \boldsymbol{\Lambda} \mathbf{E}^{H}+\frac{E_{w}}{N_{o}}\left(\mathbf{D}_{\mathbf{f}_{\mathbf{w}}} \mathbf{E}\right) \boldsymbol{\Lambda}\left(\mathbf{D}_{\mathbf{f}_{\mathbf{w}}} \mathbf{E}\right)^{H}+\mathbf{I}\right)^{-1} \mathbf{s}$.

From the last equation, it can be noted that when one of the clutter terms is dominant, the processor simplifies. When land clutter is dominant, the filter becomes

$$
\mathbf{w}_{o p t}^{1}=\frac{1}{N_{o}}\left(\frac{E_{l}}{N_{o}} \mathbf{E} \boldsymbol{\Lambda} \mathbf{E}^{H}+\mathbf{I}\right)^{-1} \mathbf{s} .
$$

Similarly when weather clutter is the dominant factor, we get

$$
\begin{aligned}
\mathbf{w}_{o p t}^{w} & =\frac{1}{N_{o}}\left(\frac{E_{w}}{N_{o}} \mathbf{D}_{\mathbf{f}_{\mathbf{w}}} \mathbf{E} \boldsymbol{\Lambda} \mathbf{E}^{H} \mathbf{D}_{\mathbf{f}_{\mathbf{w}}}^{\mathbf{H}}+\mathbf{D}_{\mathbf{f}_{\mathbf{w}}} \mathbf{D}_{\mathbf{f}_{\mathbf{w}}}^{\mathbf{H}}\right)^{-1} \mathbf{s} \\
& =\mathbf{D}_{\mathbf{f}_{\mathbf{w}}} \mathbf{w}_{o p t}^{1} \mathbf{D}_{\mathbf{f}_{\mathbf{w}}}^{\mathbf{H}}
\end{aligned}
$$

From equation (8), it can be noted the optimal filter to remove the weather clutter when land clutter is negligible, is formed by the frequency shifted version of the land clutter filter.

In many practical systems, the clutter power is estimated and stored in a clutter map. The clutter power information is used to fetch pre-calculated optimal combination weights $\left(\mathbf{w}_{\text {opt }}\right)$ from a look-up table. The storage requirements for the lookup table can be critical in many applications. The land clutter removal system whose equation is given in (7) has only one free parameter $E_{l} / N_{o}$. Therefore a look-up table with a single index (dimension) is sufficient to store the coefficients. A filter strictly removing weather clutter can be easily adapted from the stored land clutter removal filter coefficients using equation (8). But for a general system capable of removing land and weather clutter at the same time, the dimensions of storage requires changes from 1 to 3 , that is in addition to $E_{l} / N_{o}$ one has to store $E_{w} / N_{o}$ and $f_{w}$. Since the dimensions for the storage unit is tripled, the system may become infeasible to implement via pre-calculated look-up tables.

In this paper, we present alternative methods for the solution of the problem with feasible storage at the expense of some additional calculation. The additional calculations have little extra weight on the processor.

\section{Proposed Techniques}

We present three methods for the clutter suppression. The implementation complexity of these methods are briefly discussed.

\section{A. Shifted MTI Followed By Land-Only Filter}

The processing output can be defined as follows:

$$
r^{\text {out }}=\underbrace{\left(\mathbf{w}_{\text {opt }}^{l}\right)^{H} \mathbf{D}_{f_{w}}\left[\begin{array}{ccccc}
0 & 0 & \ldots & 0 & 0 \\
-1 & 1 & \ldots & 0 & 0 \\
\vdots & \vdots & & \vdots & \vdots \\
0 & 0 & \ldots & -1 & 1
\end{array}\right] \mathbf{D}_{f_{w}}^{H} \mathbf{r}}_{\left(\mathbf{w}^{(1)}\right)^{H}}
$$

With this processing, the receive vector $\mathbf{r}$ is first processed by a single order MTI filter whose null frequency is shifted to $f_{w}$ and then the land removal filter calculated from (7) is used to remove the effect of land clutter. This process can be 6) implemented by modulating the received signal by frequency $-f_{w}$, then MTI filtering, and finally by modulating it back to the frequency $f_{w}$ as shown in (9). This method is simple to implement and has minor extra computational load on the processor. The first order MTI filter described here can be generalized to higher orders, [1].

\section{B. Land Filter Followed by Weather Filter}

This method implements a cascade of land and weather clutter removal filters. For the proposed scheme, the land clutter is processed by $\mathbf{R}_{l}^{-1 / 2}$ matrix where $\mathbf{R}_{l}$ is the auto-correlation matrix of land clutter and thermal noise, $\mathbf{R}_{l}=\frac{E_{l}}{N_{o}} \mathbf{R}_{\mathbf{c}_{1}}+\mathbf{I}$. This operation corresponds to the whitening of the signal if weather clutter component is ignored. After this stage, the 
resultant vector is one more time processed with $\mathbf{R}_{\mathbf{w}}{ }^{-1 / 2}$ which is the whitening matrix for $\mathbf{r}$ in the absence of land clutter. Finally, the signal $\mathbf{s}$ is captured by matched filtering. This method approximates the whitened matched filter solution which is known to be equivalent to the SNR maximizing solution given in (3). The approximate whitened matched filter vector becomes

$$
\mathbf{w}^{(2)}=\mathbf{R}_{l}{ }^{-1 / 2} \mathbf{R}_{\mathbf{w}}{ }^{-1} \mathbf{R}_{l}^{-1 / 2} \mathbf{s}
$$

This processor requires storage of inverse of $\mathbf{R}_{l}$ and $\mathbf{R}_{\mathbf{w}}$ matrices. Since the parameter space for two matrices is decoupled the storage requirements doubles in size but not in dimension. However, the increase in computational complexity is quite significant since the computation involves multiple matrix multiplications. Alternatively the vector $\mathbf{w}^{(2)}$ in (10) can be stored to reduce the number of operations. In this case, the look-up table dimension increases by 1 . One should decide on one of these approaches based on the system specifications.

\section{Approximations to the Optimal Solution}

The equation (5) gives the optimal solution for arbitrary clutter powers and weather clutter Doppler frequency center. An approximate yet computationally feasible approach is to approximate the weather clutter correlation matrix with lower rank matrices. Since the clutter matrix is highly correlated, very few terms can be sufficient to represent the matrix accurately.

The eigen-decomposition of weather clutter is given as follows

$$
\mathbf{R}_{\mathbf{w}}=\sum_{k=1}^{N} \lambda_{k} \mathbf{u}_{k} \mathbf{u}_{k}{ }^{H}
$$

In equation (11), the vector $\mathbf{u}_{k}$ is the unit-norm eigenvector of $\mathbf{R}_{\mathbf{w}}$ corresponding to the $k^{\text {th }}$ largest eigenvalue $\lambda_{k}$. As stated in (8), the vectors $\mathbf{u}_{k}$ are modulated (frequency shifted) versions of the eigenvectors of the land clutter matrix.

In this paper we approximate $\mathbf{R}_{\mathbf{w}}$ with rank-1 and rank2 matrices and apply matrix inversion lemma ${ }^{1}$ to derive a Doppler processor. The generalization to higher order approximations is possible but not pursued here. The rank-1 and rank2 approximation for $\mathbf{R}_{\mathbf{w}}$ is given as follows

$$
\begin{aligned}
& \widehat{\mathbf{R}}_{w}^{(1)} \cong \operatorname{trace}\left(\mathbf{R}_{\mathbf{w}}\right) \mathbf{u}_{1} \mathbf{u}_{1}^{H} \\
& \widehat{\mathbf{R}}_{w}^{(2)} \cong \operatorname{trace}\left(\mathbf{R}_{\mathbf{w}}\right)\left(\frac{\lambda_{1}}{\lambda_{1}+\lambda_{2}} \mathbf{u}_{1} \mathbf{u}_{1}^{H}+\frac{\lambda_{2}}{\lambda_{1}+\lambda_{2}} \mathbf{u}_{2} \mathbf{u}_{2}^{H}\right)
\end{aligned}
$$

The approximations are weighted according to energy in each eigenvector direction. The total energy (trace) of the original $\mathbf{R}_{\mathrm{w}}$ and its approximations are set to be the same. We also note the fact that due to the normalization $\operatorname{trace}\left(\mathbf{R}_{\mathbf{w}}\right)=N$.

$$
{ }^{1}(\mathbf{A}+\mathbf{U C V})^{-1}=\mathbf{A}^{-1}-\mathbf{A}^{-1} \mathbf{U}\left(\mathbf{C}^{-1}+\mathbf{V A}^{-1} \mathbf{U}\right)^{-1} \mathbf{V A}^{-1}
$$

When the reduced rank approximations are substituted for $\mathbf{R}_{\mathrm{w}}$ in (5), we get the following

$$
\begin{aligned}
\widehat{\mathbf{w}}_{o p t}^{(1)} & =\frac{1}{N_{o}}\left(\frac{E_{l}}{N_{o}} \mathbf{R}_{\mathbf{c}_{1}}+\frac{E_{w}}{N_{o}} \widehat{\mathbf{R}}_{w}^{(1)}+\mathbf{I}\right)^{-1} \mathbf{s} \\
& =\frac{1}{N_{o}}\left(\mathbf{R}_{l}+\frac{E_{w}}{N_{o}} N \mathbf{u}_{1} \mathbf{u}_{1}^{H}\right) \mathbf{s} \\
& =\frac{1}{N_{o}}\left(\mathbf{R}_{l}^{-1}-\frac{1}{\sigma_{c}^{2}} \mathbf{c c}^{H}\right) \mathbf{s} .
\end{aligned}
$$

where $\mathbf{c}=\mathbf{R}_{l}^{-1} \mathbf{u}_{1}$ and $\sigma_{c}^{2}=N_{o} /\left(N E_{w}\right)+\mathbf{u}_{1}^{H} \mathbf{R}_{l}^{-1} \mathbf{u}_{1}$ follows from the matrix inversion lemma.

The last line of equation (14) contains two terms. The first term is $1 / N_{o} \mathbf{R}_{l}{ }^{-1} \mathbf{s}$ and it stands for the cancellation of clutter in the absence of weather clutter. The second term is the correction on the first term when the weather clutter of power $E_{w}$ coexists. It can be noted that the effect of second terms vanishes as $E_{w}$ gets smaller.

When the same process is repeated for rank-2 approximation, we get

$\widehat{\mathbf{w}}_{o p t}^{(2)}=\frac{1}{N_{o}}\left(\mathbf{R}_{l}^{-1}-C \mathbf{c} \mathbf{c}^{H}-D \mathbf{d d} \mathbf{d}^{H}+\operatorname{Ereal}\left(\mathbf{d} \mathbf{c}^{H}\right)\right) \mathbf{s}$.

The variables appearing in equation (15) are given as follows:

$$
\begin{aligned}
\mathbf{c} & =\mathbf{R}_{l}{ }^{-1} \mathbf{u}_{1} \\
\mathbf{d} & =\mathbf{R}_{l}{ }^{-1} \mathbf{u}_{2} \\
C & =1 / \sigma_{c}^{2}+|\gamma|^{2} /\left(\sigma_{c}^{4} \sigma_{d}^{2}\right) \\
D & =1 / \sigma_{d}^{2} \\
E & =\gamma /\left(\sigma_{c}^{2} \sigma_{d}^{2}\right) .
\end{aligned}
$$

The parameters linking the clutter powers to the parameters used in (15) are

$$
\begin{aligned}
\gamma & =\mathbf{u}_{2}{ }^{H} \mathbf{R}_{l}{ }^{-1} \mathbf{u}_{1} \\
\sigma_{c}^{2} & =N_{o} /\left(N E_{w}\right)\left(\lambda_{1}+\lambda_{2}\right) / \lambda_{1}+\mathbf{u}_{1}^{H} \mathbf{R}_{l}{ }^{-1} \mathbf{u}_{1} \\
\sigma_{d}^{2} & =N_{o} /\left(N E_{w}\right)\left(\lambda_{1}+\lambda_{2}\right) / \lambda_{2}+\mathbf{u}_{2}^{H} \mathbf{R}_{l}{ }^{-1} \mathbf{u}_{2}-|\gamma|^{2} / \sigma_{c}^{2} .
\end{aligned}
$$

We note that there is no additional storage requirements for the proposed solution if inverse of $\mathbf{R}_{l}$ matrices are stored in the look-up table. The vectors $\mathbf{c}$ and $\mathbf{d}$ can either be calculated on-line increasing computational complexity due to matrix multiplications; or if the online calculation is not feasible, the vectors $\mathbf{c}, \mathbf{d}$ and scalars $\mathbf{u}_{2}{ }^{H} \mathbf{R}_{l}{ }^{-1} \mathbf{u}_{1}, \mathbf{u}_{1}^{H} \mathbf{R}_{l}{ }^{-1} \mathbf{u}_{1}$ and $\mathbf{u}_{2}^{H} \mathbf{R}_{l}^{-1} \mathbf{u}_{2}$ can be pre-calculated and stored in a look-up table. All of the mentioned variables are functions of $f_{w}$ and hence the storage dimension increases only by one.

\section{NUMERICAL COMPARISONS}

In this section, we compare the performance of the methods presented in Section 3.

In Figure 1 , the improvement factor $\left(\mathrm{SNR}^{\text {out }} / \mathrm{SNR}^{\mathrm{in}}\right)$ of each system is given. In this figure, the number of processing pulses is 16, land and weather clutter both has a power of 40 $\mathrm{dB}$ above the thermal noise level, $\rho=0.999$ and the weather clutter has a Doppler frequency at $1 / 2 \mathrm{PRF}$. In Figure 1, the improvement factor (IF) for the optimal solution and 
the proposed solutions are given. The approximations to the optimal solution is given for rank-0, rank-1 and rank- 2 cases. The rank- 0 case corresponds to the case when weather clutter is completely ignored and the land clutter filter is applied as if the weather clutter does not exist.

One can note from Figure 1 that there is a significant loss of performance if the weather clutter is ignored. Shifted MTI results in some improvement and the approximate whitening method provides further improvements on MTI. The approximate optimal solutions provide close to the optimal solution for the rank-2 approximation.

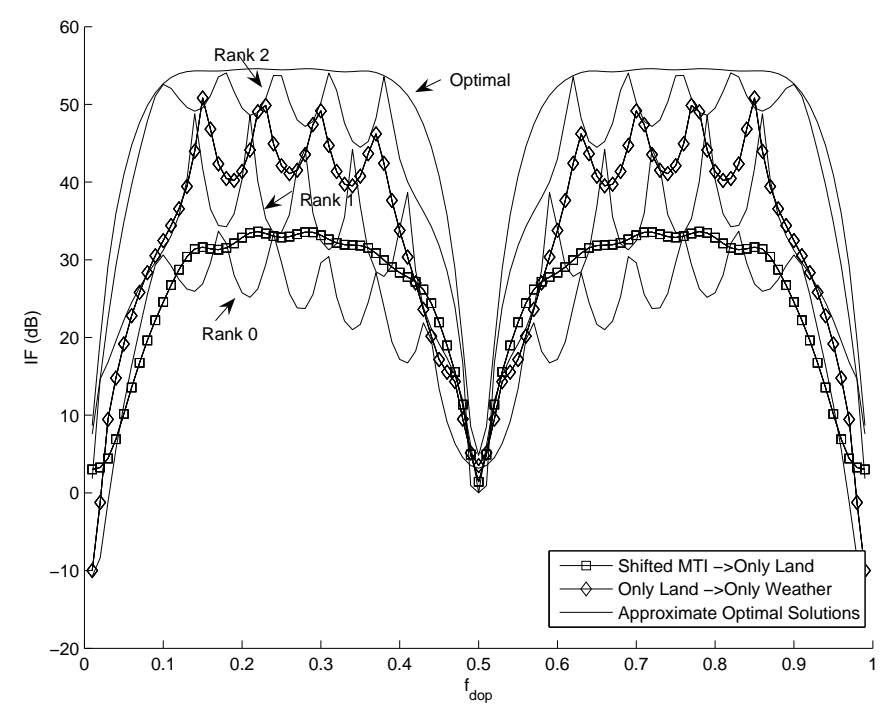

Fig. 1. $\quad N=16, E_{l} / N_{o}=40 \mathrm{~dB}, E_{w} / N_{o}=40 \mathrm{~dB}, f_{w}=0.5 \mathrm{PRF}$

In Figure 2, the performance of the methods is compared when weather clutter Doppler frequency is changed to 0.2PRF (all other parameters are the same as of Figure 1). It can be noted that the shifted MTI and the successive whitening methods have a similar performance under these conditions. The rank-2 approximation to the optimal solution is close to the optimal solution.

In Figure 3, the performance of the methods is compared when weather clutter is at $20 \mathrm{~dB}$ over $N_{o}$ (all other parameters are the same as of Figure 2). It can be noted that shifted MTI presents a poorer performance than rank-0 approximation.

A disadvantage of the shifted MTI method is that it can not adapt its attenuation to the power of weather clutter. In some scenarios such as the one presented in Figure 3, ignoring the weather clutter can be more beneficial than applying shifted MTI method. The second method which is the successive whitening method presents a performance close to optimal for this scenario. The rank-2 approximation is virtually identical to the optimal solution.

\section{CONCLUSIONS}

We have presented methods to jointly suppress land and weather clutter signals. The optimal method for clutter cancellation is known, but is not practical to implement in many

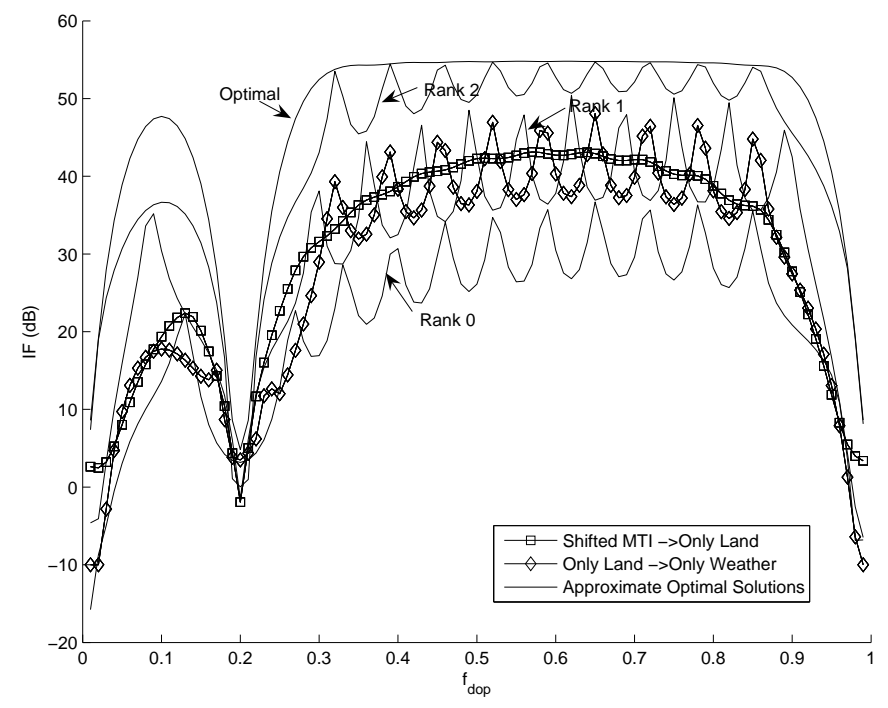

Fig. 2. $N=16, E_{l} / N_{o}=40 \mathrm{~dB}, E_{w} / N_{o}=40 \mathrm{~dB}, f_{w}=0.2 \mathrm{PRF}$

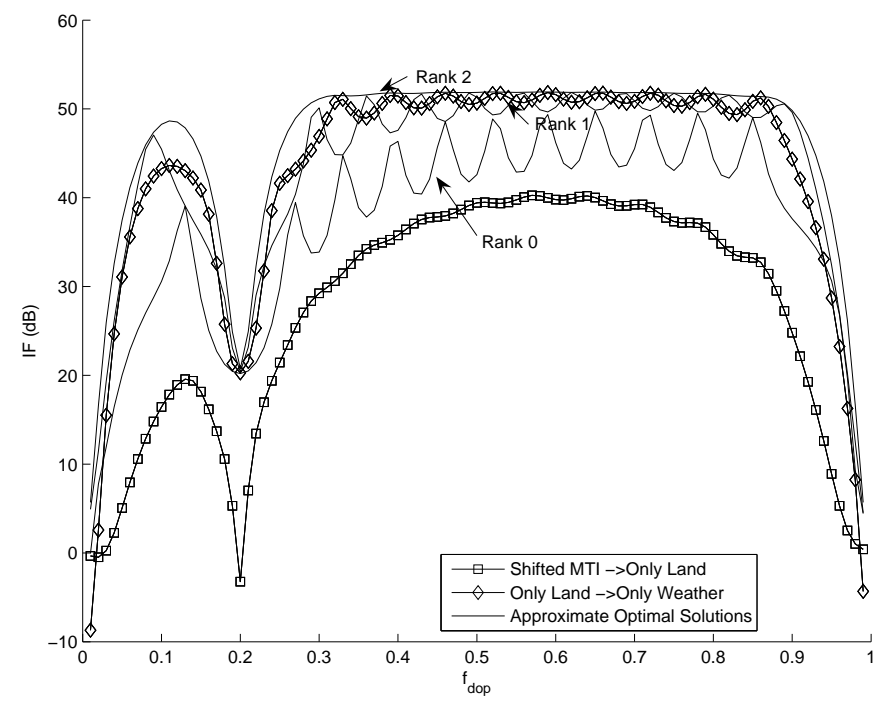

Fig. 3. $N=16, E_{l} / N_{o}=40 \mathrm{~dB}, E_{w} / N_{o}=20 \mathrm{~dB}, f_{w}=0.2 \mathrm{PRF}$

applications. In this paper, we have used rank-1 and rank-2 approximations to the weather clutter correlation matrix to define Doppler processors which are more suitable for the implementation. The proposed system can adapt its attenuation power based on the central Doppler frequency of weather clutter and the relative power levels of land - weather clutter system. The improvement over the alternative solutions has been shown to be significant in many scenarios.

\section{ACKNOWLEDGMENT}

Authors would like to acknowledge the support provided by ASELSAN A.Ş for this work. 


\section{REFERENCES}

[1] M. A. Richards, Fundamentals of Radar Signal Processing. New York: McGraw-Hill, 2005.

[2] E.DAddio, A. Farina, and F. Studer, "Performance comparison of optimum and conventional MTI and Doppler processors," IEEE Trans. on Aerospace and Electronic Systems, vol. 20, pp. 707-714, 1984.

[3] D. Schleher, "Performance comparison of MTI and coherent Doppler processors," IEEE Radar Conference, pp. 154-158, 1980.

[4] J. Hsiao, "On the optimization of MTI clutter rejection," IEEE Trans. on Aerospace and Electronic Systems, vol. 20, pp. 622-629, 1974.

[5] D. Schleher, MTI and pulsed Doppler radar. Artech House, 1991. 